\title{
THE GENERALIZED BURGERS EQUATION WITH AND WITHOUT A TIME DELAY
}

\author{
NEJIB SMAOUI AND MONA MEKKAOUI
}

Received 3 October 2002 and in revised form 27 April 2003

We consider the generalized Burgers equation with and without a time delay when the boundary conditions are periodic with period $2 \pi$. For the generalized Burgers equation without a time delay, that is, $u_{t}=v u_{x x}-u u_{x}+u+h(x), 0<x<2 \pi, t>0, u(0, t)=$ $u(2 \pi, t), u(x, 0)=u_{0}(x)$, a Lyapunov function method is used to show boundedness and uniqueness of a steady state solution and global stability of the equation. As for the generalized time-delayed Burgers equation, that is, $u_{t}(x, t)=v u_{x x}(x, t)-u(x, t-\tau) u_{x}(x, t)+$ $u(x, t), 0<x<2 \pi, t>0, u(0, t)=u(2 \pi, t), t>0, u(x, s)=u_{0}(x, s), 0<x<2 \pi,-\tau \leq s \leq 0$, we show that the equation is exponentially stable under small delays. Using a pseudospectral method, we present some numerical results illustrating and reinforcing the analytical results.

\section{Introduction}

Recently, the generalized Burgers equation

$$
u_{t}=v u_{x x}-u u_{x}+m u+h(x), \quad v, m \in \mathbb{R},
$$

has gotten a lot of attention and interest from both the engineering and the mathematical communities to model several problems including but not limited to the control of turbulent flow $[2,9]$, the excitation of long water waves by a moving pressure distribution [1], the dispersal of a population [32], and the behavior of the flame front interface under physical assumption [29]. Rakib and Sivashinsky [29] derived a nonlinear evolution equation as a model for the flame front interface:

$$
\begin{aligned}
& y_{t}-\frac{1}{2} y_{x}^{2}=v y_{x x}+y-\int_{0}^{1} y d x, \quad 0<x<1, t>0, \\
& y_{x}(0, t)=0, \quad y_{x}(1, t)=0, \quad y(x, 0)=y_{0}(x),
\end{aligned}
$$


where $\nu>0$ is a small positive constant. Later on, Sun and Ward [33] studied (1.2) by reformulating it in terms of the slope $u(x, t)=-y_{x}(x, t)$, which yields

$$
\begin{gathered}
u_{t}=v u_{x x}-u u_{x}+u, \quad 0<x<1, t>0, \\
u(0, t)=u(1, t)=0, \quad u(x, 0)=u_{0}(x) .
\end{gathered}
$$

They showed that for $v \ll 1$ with a certain class of initial conditions, the solution will have a metastable behavior. Generally, a solution is called metastable if the change of its motion can be noticed only on very long-time intervals [13].

In this paper, we study the behavior of the solution of $(1.1)$ on $[0,2 \pi]$, with periodic boundary conditions, and different values of $h$. It should be noted that the case where $m=1$ and $h=0$ in (1.1) reduces to (1.4). Also, we investigate the dynamical behavior of the generalized Burgers equation if a time delay $\tau$ is introduced in the convective term. That is,

$$
\begin{gathered}
u_{t}(x, t)=v u_{x x}(x, t)-u(x, t-\tau) u_{x}(x, t)+u(x, t), \quad 0<x<2 \pi, t>0, \\
u(0, t)=u(2 \pi, t), \quad t>0, \\
u(x, s)=u_{0}(x, s), \quad 0<x<2 \pi,-\tau \leq s \leq 0 .
\end{gathered}
$$

The effect of time delays in PDEs has been studied by different investigators to see whether or not such delays can destabilize the system under study $[10,11,12,14,16,17,25,26$, 27,28 ]. Oliva [28] is one of the investigators who considered dissipative scalar reactiondiffusion equations with boundary conditions which include small delays. He showed the global existence and uniqueness of solution in a convenient fractional power space. Also, Datko [10] and Datko et al. [11, 12] studied certain hyperbolic partial differential equations with Neumann boundary conditions that include time delays. These equations are the Euler beam equation and the two-dimensional wave equation on a square. They showed that these equations could be destabilized when small delays are introduced into their boundary feedback controls. However, Friesecke [17] considered Hutchinson's equation which arises in population dynamics as a model for the evolution of a population with density distribution $u$. He studied equations of the following form:

$$
u_{t}-\mu \Delta u=f(u(t), u(t-\tau))
$$

and showed that all nonnegative solutions of the initial boundary value problem stay bounded as $t \rightarrow \infty$ in one, two, or more dimensions. Burgers equation with time delays was also investigated by Liu [25] who considered the following form of Burgers equation:

$$
u_{t}(x, t)=v u_{x x}(x, t)-u(x, t-\tau) u_{x}(x, t), \quad 0<x<1, t>0,
$$

with Dirichlet boundary condition. He showed that the delayed Burgers equation is exponentially stable if the delay parameter is sufficiently small.

This paper is organized as follows. Section 2 analyzes the behavior of the solution of (1.1) without introducing a time delay. Section 3 discusses the behavior of the timedelayed Burgers equation (1.6). Section 4 presents some numerical results for both studies that support the analytical results, and we conclude in Section 5. 


\section{The generalized Burgers equation without a time delay}

The forced Burgers equation without a time delay has been the subject of numerous studies $[1,4,5,6,7,8,9,15,16,19,20,21,22,30,31]$. Ablowitz and De Lillo [1] considered Burgers equation

$$
u_{t}=\left(u_{x}+u^{2}\right)_{x}+G(x, t)
$$

where $u=u(x, t)$ and $G(x, t)$ is a given function. They linearized the initial value problem on the line for an integrable bounded function of time $F(t)$ and discussed the asymptotic behavior of the solution for particular choices of $F(t)$. Ito and Yan [20] studied the forced viscous scalar conservation law on $(0,1)$ with the nonlinear flux feedback at the boundary

$$
u_{t}=v u_{x x}+u u_{x}+F(x, t), \quad x \in(0,1), t>0
$$

They showed that under an appropriate growth condition on the flux function and nonlinear dissipation at the boundary, there exists an absorbing set that absorbs the whole space $L^{\infty}(0,1)$, and they proved the existence of a compact global attractor in the $L^{\infty}$ topology.

Smaoui [31] studied the long-time dynamics of a system of reaction-diffusion equations that arise from the viscous forced Burgers equation where the force is sinusoidal,

$$
u_{t}=v u_{x x}-u u_{x}+F(x)
$$

with periodic boundary conditions. He used a nonlinear transformation introduced by Kwak to embed the scalar Burgers equation into a system of reaction-diffusion equations. He showed analytically as well as numerically that the two systems have a similar longtime dynamical behavior for large viscosity $\nu$.

2.1. $h(x)=0$. In the following, we show that the generalized Burgers equation when $h(x)=0$,

$$
u_{t}=v u_{x x}-u u_{x}+u
$$

and with periodic boundary condition, is globally stable. Let

$$
V(t)=\frac{1}{2} \int_{0}^{2 \pi} u^{2} d x
$$

then

$$
\frac{d V}{d t}=\frac{d}{d t}\left(\frac{1}{2} \int_{0}^{2 \pi} u^{2} d x\right)=\int_{0}^{2 \pi} u u_{t} d x=\int_{0}^{2 \pi} u\left(\nu u_{x x}-u u_{x}+u\right) d x
$$

Using integration by parts and the periodicity of $u$, we get

$$
\frac{d}{d t}\left(\frac{1}{2} \int_{0}^{2 \pi} u^{2} d x\right)=-v \int_{0}^{2 \pi} u_{x}^{2} d x+\int_{0}^{2 \pi} u^{2} d x
$$


Applying the Poincare inequality and the zero-mean condition on $u$ on the above equation, we obtain

$$
\frac{d}{d t}\left(\frac{1}{2} \int_{0}^{2 \pi} u^{2} d x\right) \leq-\left(\frac{v}{4 \pi^{2}}-1\right) \int_{0}^{2 \pi} u^{2} d x \leq 0, \quad \forall v \geq 4 \pi^{2} .
$$

By Lyapunov theory, $\lim _{t \rightarrow \infty} u(x, t)=0$, which implies that $(2.4)$ is globally asymptotically stable.

Global stability can also be shown by using control theory. Let $u^{s}$ be the steady state solution of $u$, then

$$
\lim _{t \rightarrow \infty} u(x, t)=u^{s}, \quad \forall x \in[0,2 \pi]
$$

If one defines the regulation error by

$$
w(x, t)=u(x, t)-u^{s},
$$

then (2.4) becomes

$$
w_{t}=\nu w_{x x}-\left(w+u_{s}\right) w_{x}+w+u^{s}=v w_{x x}-w w_{x}-u_{s} w_{x}+w+u^{s},
$$

with periodic boundary control $w(0, t)=f_{0}$, and $w(2 \pi, t)=f_{1}$, where $f_{0}$ and $f_{1}$ are scalar control inputs. Then by taking the control Lyapunov function

$$
V=\frac{1}{2} \int_{0}^{2 \pi} w^{2}(x, t) d x
$$

and taking the time derivative of $V$, one can then find the control $f_{0}$ and $f_{1}$ that can enhance the negativity of $(d / d t)(V)$ which implies that $w(x, t)=0$ or $u(x, t)=u^{s}$. Thus, (2.4) is globally asymptotically stable in $L_{2}(0,2 \pi)$. (For a complete stability analysis using control theory, the reader is referred to $[3,9,19,23,24,26,27]$.)

2.2. $h(x) \neq 0$. In this section, we show that in the Hilbert space $H=H^{2}(0,2 \pi)$ consisting of $2 \pi$-periodic functions with zero mean, the first and second derivatives in $L^{2}(0,2 \pi)$, and inner product $\langle u, v\rangle_{2}=\int u_{x x} v_{x x} d x$, the generalized Burgers equation

$$
u_{t}=v u_{x x}-u u_{x}+u+h(x)
$$

with periodic boundary condition $u(0, t)=u(2 \pi, t)$, has a unique steady state solution.

Proposition 2.1. Every solution to the generalized Burgers equation

$$
u_{t}=v u_{x x}-u u_{x}+u+h(x)
$$

satisfies the inequality

$$
\|u\| \leq \sqrt{\frac{2 \pi^{2}}{v-8 \pi^{2}}}\|h\|, \quad \forall t \geq t_{0}
$$


with

$$
t_{0}=\frac{4 \pi^{2}}{v-8 \pi^{2}} \ln \left(\frac{v\left\|u_{0}\right\|^{2}}{2 \pi^{2}\|h\|^{2}}\right), \quad v>8 \pi^{2}
$$

Proof. Similar to [32, Theorem 4.1].

Proposition 2.2. Let $v>8 \pi^{2}$ and let $u^{s}$ be the steady state solution to the forced Burgers equation, then $u^{s}$ satisfies the following inequalities:

(a) $\left\|u^{s}\right\| \leq \sqrt{16 \pi^{4} / \nu\left(\nu-8 \pi^{2}\right)}\|h\|$,

(b) $\left\|u_{x}^{s}\right\| \leq c\|h\|$, where $c=\left(4 \pi^{2} /\left(\nu-4 \pi^{2}\right) \sqrt{\nu\left(\nu-8 \pi^{2}\right)}\right)^{1 / 2}$.

Proof. Similar to [31, Lemma 3.5].

Theorem 2.3. Let $c_{1}$ be the Sobolev constant and let $c=\left(4 \pi^{2} /\left(\nu-4 \pi^{2}\right) \sqrt{\nu\left(\nu-8 \pi^{2}\right)}\right)^{1 / 2}$ with $v>8 \pi^{2}$. The generalized Burgers equation

$$
u_{t}=v u_{x x}-u u_{x}+u+h
$$

has a unique steady state solution provided

$$
\|h\|<\left(\frac{\nu-4 \pi^{2} c_{1}}{6 \pi^{2} c c_{1}}\right)
$$

Proof. Suppose there are two steady state solution $u$ and $v$ such that

$$
v u_{x x}-u u_{x}+u+h(x)=0, \quad v v_{x x}-v v_{x}+v+h(x)=0 .
$$

Let $w=u-v$. Then,

$$
\nu w_{x x}-u w_{x}-v_{x} w+w=0 .
$$

Multiplying the above equation by $w$, integrating from 0 to $2 \pi$, and using the periodicity of $u$ and $w$ lead to

$$
\nu \int_{0}^{2 \pi} w_{x}^{2} d x+\int_{0}^{2 \pi} u\left(\frac{w^{2}}{2}\right)_{x} d x+\int_{0}^{2 \pi} v_{x} w^{2} d x-\int_{0}^{2 \pi} w^{2} d x=0
$$

Again, using integration by parts on $\int_{0}^{2 \pi} u\left(w^{2} / 2\right)_{x} d x$, we obtain

$$
v \int_{0}^{2 \pi} w_{x}^{2} d x-\int_{0}^{2 \pi} w^{2}\left(\frac{u_{x}}{2}-v_{x}+1\right) d x=0 .
$$

Equation (2.22) can be written as

$$
v\left\|w_{x}\right\|^{2} \leq\left\|w^{2}\right\|\left(\frac{1}{2}\left\|u_{x}\right\|+\left\|v_{x}\right\|+1\right)
$$


Now using part (b) of Proposition 2.2, we obtain

$$
\nu\left\|w_{x}\right\|^{2} \leq\left\|w^{2}\right\|\left(\frac{c}{2}\|h\|+c\|h\|+1\right)
$$

or

$$
\left\|w_{x}\right\|^{2} \leq\left\|w^{2}\right\|\left(\frac{3 c}{2 v}\|h\|+\frac{1}{v}\right)
$$

Since

$$
\left\|w^{2}\right\|_{L^{2}(0,2 \pi)} \leq c_{1}\|w\|_{L^{\infty}(0,2 \pi)}^{2} \leq 2 \pi c_{1}\|w\|_{L^{2}(0,2 \pi)}\left\|w_{x}\right\|_{L^{2}(0,2 \pi)},
$$

it follows that

$$
\left\|w_{x}\right\|^{2} \leq\left(\frac{3 c}{2 v}\|h\|+\frac{1}{v}\right)\left(2 \pi c_{1}\|w\|\left\|w_{x}\right\|\right)
$$

or

$$
\left\|w_{x}\right\|^{2} \leq\left(\frac{6 \pi^{2} c c_{1}}{v}\|h\|+\frac{4 \pi^{2} c_{1}}{v}\right)\left\|w_{x}\right\|^{2}
$$

If

$$
\left(\frac{6 \pi^{2} c c_{1}}{v}\right)\|h\|+\frac{4 \pi^{2} c_{1}}{v} \leq 1
$$

or

$$
\|h\|<\frac{\nu-4 \pi^{2} c_{1}}{6 \pi^{2} c c_{1}}
$$

then $w=w_{x}=0$, which implies $u=v$.

\section{The generalized Burgers equation with a time delay}

The effect of time delays in certain partial differential equations has been the subject of recent studies $[10,11,12,14,16,17,25,26,27,28]$. The question that is frequently asked is: can such delays destabilize a system which is stable in the absence of delays? Oliva [28] considered dissipative scalar reaction-diffusion equations that include the ones of the form

$$
u_{t}-\Delta u=f(u(t))
$$

subjected to boundary conditions that include small delays

$$
\frac{\partial u}{\partial n_{a}}=g(u(t), u(t-\tau))
$$

He proved the global existence and uniqueness of solutions in a convenient fractional power space. Furthermore, he showed that, for $\tau$ sufficiently small, all bounded solutions 
are asymptotic to the set of equilibria as $t$ tends to infinity. On the other hand, Liu [25] considered the time-delayed Burgers equation

$$
u_{t}(x, t)=v u_{x x}(x, t)-u(x, t-\tau) u_{x}(x, t), \quad 0<x<1, t>0,
$$

with Dirichlet boundary condition. He showed that the delayed Burgers equation is exponentially stable if the delay parameter is sufficiently small.

In this section, the generalized Burgers equation with time delay is studied:

$$
\begin{gathered}
u_{t}(x, t)=v u_{x x}(x, t)-u(x, t-\tau) u_{x}(x, t)+u(x, t), \quad 0<x<2 \pi, t>0, \\
u(0, t)=u(2 \pi, t), \quad t>0, \\
u(x, s)=u_{0}(x, s), \quad 0<x<2 \pi,-\tau \leq s \leq 0 .
\end{gathered}
$$

First, we show that the problem given by (3.4), (3.5), and (3.6) is well posed. We define the linear operator $A$ by

$$
\begin{gathered}
A: H^{2}(0,2 \pi) \longrightarrow L^{2}(0,2 \pi) \\
A w=v w_{x x}+w
\end{gathered}
$$

where $H^{2}(0,2 \pi)$ consists of $2 \pi$-periodic functions with zero mean, two derivatives in $L^{2}(0,2 \pi)$, and inner product $\langle u, v\rangle_{2}=\int u_{x x} v_{x x} d x$. It is well known that the operator $A$ generates an analytic semigroup $e^{A t}$ in $L^{2}(0,2 \pi)$ (see Temam [34]). Also, we define the nonlinear operator

$$
\begin{gathered}
B: C\left([-\tau, 0], H^{1}(0,2 \pi)\right) \longrightarrow L^{2}(0,2 \pi), \\
B(\varphi)=-\varphi(-\tau) \varphi_{x}(0),
\end{gathered}
$$

where $B$ is locally Lipschitz. If we denote

$$
u^{t}(s)=u(t+s), \quad-\tau \leq s \leq 0,
$$

then the generalized Burgers equation (3.4) can be written in terms of the above operators as

$$
u_{t}=A u+B\left(u^{t}(s)\right)
$$

Using Gronwall's inequality, we obtain

$$
\begin{gathered}
u(t)=e^{A t} u_{0}(0)+\int_{0}^{t} e^{A(t-s)} B\left(u^{s}\right) d s, \quad t>0, \\
u(t)=u_{0}(t), \quad-\tau \leq t \leq 0
\end{gathered}
$$


Lemma 3.1. The generalized Burgers equation

$$
\begin{gathered}
u_{t}(x, t)=v u_{x x}(x, t)-u(x, t-\tau) u_{x}(x, t)+u(x, t), \quad 0<x<2 \pi, t>0, \\
u(0, t)=u(2 \pi, t), \quad t>0, \\
u(x, s)=u_{0}, \quad 0<x<2 \pi,-\tau \leq s \leq 0,
\end{gathered}
$$

with $u_{0}=u_{0}(x, s) \in C\left([-\tau, 0], H^{1}(0,2 \pi)\right)$, has a unique global solution $u$ on $[-\tau, \infty)$ with

$$
u \in C\left([-\tau, \infty), H^{1}(0,2 \pi)\right) .
$$

Proof. See [28, Theorem 1].

In the following, we show that (3.12) does not blow up for finite time. Let $n \tau \leq t \leq$ $(n+1) \tau(n=0,1, \ldots)$. First, we prove that for $n=0$ and for any $\tau \geq 0,(3.12)$ does not blow up for finite time. Then, we use continuation to show that this is true for all $n$. For $n=0$ (i.e., $0 \leq t \leq \tau$ ),

$$
\frac{d}{d t} \int_{0}^{2 \pi} u_{x}^{2}(t) d x=2 \int_{0}^{2 \pi} u_{x}(t) u_{x t}(t) d x
$$

Using integration by parts on the right-hand side of (3.16) and making use of the periodicity of $u$, we get

$$
\begin{aligned}
\frac{d}{d t} \int_{0}^{2 \pi} u_{x}^{2}(t) d x & =-2 \int_{0}^{2 \pi} u_{x x}(t) u_{t}(t) d x \\
& =-2 v \int_{0}^{2 \pi} u_{x x}^{2}(t) d x+2 \int_{0}^{2 \pi} u(t-\tau) u_{x}(t) u_{x x}(t) d x-2 \int_{0}^{2 \pi} u_{x x}(t) u(t) d x
\end{aligned}
$$

Now using the fact that $|u(t-\tau)| \leq\left\|u_{0}\right\|_{C\left([-\tau, 0], H^{1}(0,2 \pi)\right)}$, for $0 \leq t \leq \tau$, and integrating by parts the last term of the right-hand side, then the above equation becomes

$$
\begin{aligned}
\frac{d}{d t} \int_{0}^{2 \pi} u_{x}^{2}(t) d x \leq & -2 \nu \int_{0}^{2 \pi} u_{x x}^{2}(t) d x \\
& +2\left\|u_{0}\right\|_{C\left([-\tau, 0], H^{1}(0,2 \pi)\right)} \int_{0}^{2 \pi}\left|u_{x}(t) u_{x x}(t)\right| d x+2 \int_{0}^{2 \pi} u_{x}^{2}(t) d x
\end{aligned}
$$

or

$$
\begin{aligned}
& \frac{d}{d t} \int_{0}^{2 \pi} u_{x}^{2}(t) d x-2 \int_{0}^{2 \pi} u_{x}^{2}(t) d x \\
& \leq-2 v \int_{0}^{2 \pi} u_{x x}^{2}(t) d x \\
& \quad+\left\|u_{0}\right\|_{C\left([-\tau, 0], H^{1}(0,2 \pi)\right)} \int_{0}^{2 \pi} \sqrt{\frac{2 \nu}{\left\|u_{0}\right\|_{C\left([-\tau, 0], H^{1}(0,2 \pi)\right)}}} u_{x x}(t) \sqrt{\frac{\left\|u_{0}\right\|_{C\left([-\tau, 0], H^{1}(0,2 \pi)\right)}}{2 v}} u_{x}(t) d x .
\end{aligned}
$$


Next, using Cauchy-Schwarz and Young's inequalities on the above equation, we get

$$
\begin{array}{ll}
\frac{d}{d t} \int_{0}^{2 \pi} u_{x}^{2}(t) d x-2 \int_{0}^{2 \pi} u_{x}^{2}(t) d x & \\
\leq-2 \nu \int_{0}^{2 \pi} u_{x x}^{2} d x+2\left\|u_{0}\right\|_{C\left([-\tau, 0], H^{1}(0,2 \pi)\right)} & {\left[\int_{0}^{2 \pi} \frac{2 \nu}{\left\|u_{0}\right\|_{C\left([-\tau, 0], H^{1}(0,2 \pi)\right)}} \frac{u_{x x}^{2}(t)}{2} d x\right.} \\
& \left.+\int_{0}^{2 \pi} \frac{\left\|u_{0}\right\|_{C\left([-\tau, 0], H^{1}(0,2 \pi)\right)}}{2 \nu} \frac{u_{x}^{2}(t)}{2} d x\right] \\
\leq \frac{1}{2 \nu}\left\|u_{0}\right\|_{C\left([-\tau, 0], H^{1}(0,2 \pi)\right)}^{2} \int_{0}^{2 \pi} u_{x}^{2}(t) d x & \\
\leq \frac{1}{v}\left\|u_{0}\right\|_{C\left([-\tau, 0], H^{1}(0,2 \pi)\right)}^{2} \int_{0}^{2 \pi} u_{x}^{2}(t) d x, &
\end{array}
$$

which implies that

$$
\int_{0}^{2 \pi} u_{x}^{2}(t) d x \leq \exp \left(\left(\frac{1}{\nu}\left\|u_{0}\right\|_{C\left([-\tau, 0], H^{1}(0,2 \pi)\right)}^{2}+2\right) t\right) \int_{0}^{2 \pi} u_{x}^{2}(0) d x .
$$

The same result can be shown for $n \tau \leq t \leq(n+1) \tau(n=1,2, \ldots)$ by applying the same procedure. Thus, for any $\tau>0$, the solution will not blow up in a finite time.

Before stating the main result about the exponential stability, the following notations are introduced. For a given initial condition $u_{0}=u_{0}(x, s) \in C\left([-\tau, 0], H^{1}(0,2 \pi)\right)$, let

$$
\begin{aligned}
K= & K\left(u_{0}\right) \\
= & \sup _{-\tau \leq s \leq 0}\left\|u_{0_{x}}(s)\right\|+\sqrt{4\left[\left\|u_{0}(0)\right\|^{2}+\left\|u_{0_{x}}(0)\right\|^{2}\right] \exp \left[\frac{8 \pi^{4}}{v}\left(\left\|u_{0_{x}}\right\|_{L_{\tau}^{2}}^{2}+\left\|u_{0}(0)\right\|^{2}\right)\right]}, \\
\sigma= & \sigma\left(\nu, u_{0}\right) \\
= & \sup \left\{\delta>0:\left[\frac{\omega}{\omega-2}\left\|u_{0}(0)\right\|^{2}+\left\|u_{0_{x}}(0)\right\|^{2}\right] \exp \left[\frac{8 \pi^{4}}{v} e^{\omega \tau}\left(\left\|u_{0_{x}}\right\|_{L_{\tau}^{2}}^{2}+\frac{1}{\omega}\left\|u_{0}(0)\right\|^{2}\right)\right]\right. \\
& \left.\leq \frac{K^{2}}{4} \text { for } 0 \leq \tau \leq \delta\right\},
\end{aligned}
$$

where $\|\cdot\|$ denotes the $L^{2}$-norm and $\left\|u_{0_{x}}\right\|_{L_{\tau}^{2}}^{2}=\int_{-\tau}^{0} \int_{0}^{2 \pi} u_{0_{x}}^{2}(s) d x d s, \mu=\nu-4 \pi^{2}$, with $\nu>$ $4 \pi^{2}$

$$
\begin{gathered}
\tau_{0}=\tau_{0}\left(\mu, u_{0}\right)=\min \left\{\sigma,\left(\frac{-1+\sqrt{5}}{32 \pi^{4} K^{2}}\right) \mu\right\}, \\
\omega=\omega(\mu, \tau, K)=\frac{\mu-\sqrt{16 \pi^{4} \tau\left(\mu K^{2}+16 \pi^{4} \tau K^{4}\right)}}{4 \pi^{2}}>0, \quad \text { for } 0 \leq \tau \leq \tau_{0}, \mu>0 .
\end{gathered}
$$

In (3.24), $\omega>0$ because

$$
\mu-\sqrt{16 \pi^{4} \tau\left(\mu K^{2}+16 \pi^{4} \tau K^{4}\right)}>0,
$$


which is true only if

$$
-\left(\frac{1+\sqrt{5}}{32 \pi^{4} K^{2}}\right) \mu<\tau<\left(\frac{-1+\sqrt{5}}{32 \pi^{4} K^{2}}\right) \mu .
$$

Lemma 3.2. If $\left\|u_{x}(t)\right\| \leq K$, for all $-\tau \leq t<T_{0}$ with $T_{0}=\sup \left\{\delta:\left\|u_{x}(t)\right\| \leq K\right.$ on $0 \leq t \leq$ $\delta\}$, then

$$
\int_{t-\tau}^{t} \int_{0}^{2 \pi} u_{s}^{2}(s) d x d s \leq \mu K^{2}+16 \pi^{4} \tau K^{4}, \quad \forall 0 \leq t \leq T_{0} .
$$

Proof. Since

$$
\nu \frac{d}{d t} \int_{0}^{2 \pi} u_{x}^{2}(t) d x=2 v \int_{0}^{2 \pi} u_{x}(t) u_{x t}(t) d x,
$$

then integrating by parts and using the periodicity of $u$, we get

$$
\begin{aligned}
\nu \frac{d}{d t} \int_{0}^{2 \pi} u_{x}^{2}(t) d x & =-2 v \int_{0}^{2 \pi} u_{x x}(t) u_{t}(t) d x \\
& =-2 \int_{0}^{2 \pi}\left[u_{t}(t)+u(t-\tau) u_{x}(t)-u(t)\right] u_{t}(t) d x \\
& =-2 \int_{0}^{2 \pi} u_{t}^{2}(t) d x-2 \int_{0}^{2 \pi} u(t-\tau) u_{x}(t) u_{t}(t) d x+\frac{d}{d t} \int_{0}^{2 \pi} u^{2}(x, t) d x
\end{aligned}
$$

Using the Poincaré inequality and the zero-mean condition on $u$, we get

$$
\begin{aligned}
\nu \frac{d}{d t} \int_{0}^{2 \pi} u_{x}^{2}(t) d x \leq & -2 \int_{0}^{2 \pi} u_{t}^{2}(t) d x-2 \int_{0}^{2 \pi} u(t-\tau) u_{x}(t) u_{t}(t) d x \\
& +\frac{d}{d t}\left(4 \pi^{2} \int_{0}^{2 \pi} u_{x}^{2}(x, t) d x\right)
\end{aligned}
$$

Integrating (3.30) from $t-\tau$ to $t$, we get

$$
\begin{aligned}
\left(\nu-4 \pi^{2}\right) \int_{t-\tau}^{t} d( & \left.\int_{0}^{2 \pi} u_{x}^{2}(t) d x\right) \\
& \leq-2 \int_{t-\tau}^{t} \int_{0}^{2 \pi} u_{s}^{2}(s) d x d s-2 \int_{t-\tau}^{t} \int_{0}^{2 \pi} u(s-\tau) u_{x}(s) u_{s}(s) d x d s .
\end{aligned}
$$

The above equation can be written as

$$
\begin{aligned}
\mu \int_{0}^{2 \pi} u_{x}^{2}(t) d x-\mu \int_{0}^{2 \pi} & u_{x}^{2}(t-\tau) d x \\
& \leq-2 \int_{t-\tau}^{t} \int_{0}^{2 \pi} u_{s}^{2}(s) d x d s-2 \int_{t-\tau}^{t} \int_{0}^{2 \pi} u(s-\tau) u_{x}(s) u_{s}(s) d x d s .
\end{aligned}
$$


From Holder's inequality, we have $|u(s-\tau)| \leq(2 \pi)^{2}\left\|u_{x}(s-\tau)\right\| \leq 4 \pi^{2} K$ when $0 \leq x \leq$ $2 \pi$ and $-\tau \leq s<T_{0}$. Then, for $0 \leq t \leq T_{0}$,

$$
\mu \int_{0}^{2 \pi} u_{x}^{2}(t) d x+2 \int_{t-\tau}^{t} \int_{0}^{2 \pi} u_{s}^{2}(s) d x d s \leq \mu K^{2}+8 \pi^{2} K \int_{t-\tau}^{t} \int_{0}^{2 \pi} u_{x}(s) u_{s}(s) d x d s
$$

Using the Cauchy-Schwarz inequality on (3.33), we get

$$
\begin{aligned}
\mu \int_{0}^{2 \pi} & u_{x}^{2}(t) d x+2 \int_{t-\tau}^{t} \int_{0}^{2 \pi} u_{s}^{2}(s) d x d s \\
& \leq \mu K^{2}+2\left[\left(\left(4 \pi^{2} K\right)^{2} \int_{t-\tau}^{t} \int_{0}^{2 \pi} u_{x}^{2}(s) d x d s\right)^{1 / 2}\left(\int_{t-\tau}^{t} \int_{0}^{2 \pi} u_{s}^{2}(s) d x d s\right)^{1 / 2}\right] .
\end{aligned}
$$

Now using Young's inequality on the above equation, we get

$$
\begin{aligned}
& \mu \int_{0}^{2 \pi} u_{x}^{2}(t) d x+2 \int_{t-\tau}^{t} \int_{0}^{2 \pi} u_{s}^{2}(s) d x d s \\
& \quad \leq \mu K^{2}+2\left[\left(4 \pi^{2} K\right)^{2} \int_{t-\tau}^{t} \int_{0}^{2 \pi} \frac{u_{x}^{2}(s)}{2} d x d s+\int_{t-\tau}^{t} \int_{0}^{2 \pi} \frac{u_{s}^{2}(s)}{2} d x d s\right] \\
& \quad \leq \mu K^{2}+16 \pi^{4} \tau K^{4}+\int_{t-\tau}^{t} \int_{0}^{2 \pi} u_{s}^{2}(s) d x d s
\end{aligned}
$$

which implies that

$$
\int_{t-\tau}^{t} \int_{0}^{2 \pi} u_{s}^{2}(s) d x d s \leq \mu K^{2}+16 \pi^{4} \tau K^{4}-\mu \int_{0}^{2 \pi} u_{x}^{2}(t) d x
$$

or

$$
\int_{t-\tau}^{t} \int_{0}^{2 \pi} u_{s}^{2}(s) d x d s \leq \mu K^{2}+16 \pi^{4} \tau K^{4}, \quad \forall 0 \leq t \leq T_{0}
$$

Lemma 3.3. If $\left\|u_{x}(t)\right\| \leq K$, for all $-\tau \leq t<T_{0}$ and $T_{0}=\sup \left\{\delta:\left\|u_{x}(t)\right\| \leq K\right.$ on $0 \leq t \leq$ $\delta\}$, then

$$
\int_{0}^{T_{0}} e^{\omega t} \int_{0}^{2 \pi} u_{x}^{2}(t) d x d t \leq \frac{1}{\omega} \int_{0}^{2 \pi} u_{0}^{2}(x, 0) d x
$$

where $\omega$ is defined as in (3.24).

Proof.

$$
\frac{d}{d t} \int_{0}^{2 \pi} u^{2}(t) d x=2 \int_{0}^{2 \pi} u(t)\left[\nu u_{x x}(t)-u(t-\tau) u_{x}(t)+u(t)\right] d x
$$


or

$$
\frac{d}{d t} \int_{0}^{2 \pi} u^{2}(t) d x=2 v \int_{0}^{2 \pi} u(t) u_{x x}(t) d x-2 \int_{0}^{2 \pi} u(t) u(t-\tau) u_{x}(t) d x+2 \int_{0}^{2 \pi} u^{2}(t) d x
$$

Using integration by parts and the periodicity of $u$, we get

$$
\frac{d}{d t} \int_{0}^{2 \pi} u^{2}(t) d x=-2 v \int_{0}^{2 \pi} u_{x}^{2}(t) d x-2 \int_{0}^{2 \pi} u(t) u(t-\tau) u_{x}(t) d x+2 \int_{0}^{2 \pi} u^{2}(t) d x
$$

By using the Poincaré inequality and the zero-mean condition on $u$, we have

$$
\frac{d}{d t} \int_{0}^{2 \pi} u^{2}(t) d x \leq\left(-2 \nu+8 \pi^{2}\right) \int_{0}^{2 \pi} u_{x}^{2}(t) d x-2 \int_{0}^{2 \pi} u(t) u(t-\tau) u_{x}(t) d x
$$

and since $\int_{0}^{2 \pi} u^{2}(t) u_{x}(t) d x=0$ because of the periodicity of $u$, then adding that term in the above inequality, we obtain

$$
\begin{aligned}
\frac{d}{d t} \int_{0}^{2 \pi} u^{2}(t) d x \leq & \left(-2 v+8 \pi^{2}\right) \int_{0}^{2 \pi} u_{x}^{2}(t) d x \\
& -2 \int_{0}^{2 \pi} u(t) u(t-\tau) u_{x}(t) d x+2 \int_{0}^{2 \pi} u^{2}(t) u_{x}(t) d x \\
= & \left(-2 v+8 \pi^{2}\right) \int_{0}^{2 \pi} u_{x}^{2}(t) d x-2 \int_{0}^{2 \pi}[u(t-\tau)-u(t)] u(t) u_{x}(t) d x
\end{aligned}
$$

Using the fact that $|u(x, t)| \leq(2 \pi)^{2}\left\|u_{x}(t)\right\|,(3.43)$ becomes

$$
\begin{aligned}
\frac{d}{d t} \int_{0}^{2 \pi} u^{2}(t) d x \leq & \left(-2 \nu+8 \pi^{2}\right) \int_{0}^{2 \pi} u_{x}^{2}(t) d x \\
& +8 \pi^{2}\left(\int_{0}^{2 \pi} u_{x}^{2}(t) d x\right)^{1 / 2} \int_{0}^{2 \pi}\left|(u(t-\tau)-u(t)) u_{x}(t)\right| d x
\end{aligned}
$$

Now using the Cauchy-Schwarz inequality, we obtain

$$
\begin{aligned}
\frac{d}{d t} \int_{0}^{2 \pi} u^{2}(t) d x \leq & \left(-2 \nu+8 \pi^{2}\right) \int_{0}^{2 \pi} u_{x}^{2}(t) d x \\
& +8 \pi^{2}\left(\int_{0}^{2 \pi} u_{x}^{2}(t) d x\right)^{1 / 2}\left(\int_{0}^{2 \pi}|u(t-\tau)-u(t)|^{2} d x\right)^{1 / 2}\left(\int_{0}^{2 \pi} u_{x}^{2}(t) d x\right)^{1 / 2} \\
= & \left(-2 \nu+8 \pi^{2}\right) \int_{0}^{2 \pi} u_{x}^{2}(t) d x+8 \pi^{2} \int_{0}^{2 \pi} u_{x}^{2}(t) d x\left(\int_{0}^{2 \pi}|u(t-\tau)-u(t)|^{2} d x\right)^{1 / 2}
\end{aligned}
$$




$$
\begin{aligned}
& =\left(-2 v+8 \pi^{2}\right) \int_{0}^{2 \pi} u_{x}^{2}(t) d x+8 \pi^{2} \int_{0}^{2 \pi} u_{x}^{2}(t) d x\left(\int_{0}^{2 \pi}\left|\int_{t-\tau}^{t} u_{s}(s) d s\right|^{2} d x\right)^{1 / 2} \\
& =\left(-2 \nu+8 \pi^{2}\right) \int_{0}^{2 \pi} u_{x}^{2}(t) d x+8 \pi^{2} \sqrt{\tau} \int_{0}^{2 \pi} u_{x}^{2}(t) d x\left(\int_{0}^{2 \pi} \int_{t-\tau}^{t} u_{s}^{2}(s) d s d x\right)^{1 / 2} .
\end{aligned}
$$

Now using Lemma 3.2, we get

$$
\begin{aligned}
\frac{d}{d t} \int_{0}^{2 \pi} u^{2}(t) d x & \leq\left(-2 v+8 \pi^{2}\right) \int_{0}^{2 \pi} u_{x}^{2}(t) d x+8 \pi^{2} \sqrt{\tau} \sqrt{\mu K^{2}+16 \pi^{4} \tau K^{4}} \int_{0}^{2 \pi} u_{x}^{2}(t) d x \\
& \leq-2\left(\left(\nu-4 \pi^{2}\right)-\sqrt{16 \pi^{4} \tau\left(\mu K^{2}+16 \pi^{4} \tau K^{4}\right)}\right) \int_{0}^{2 \pi} u_{x}^{2}(t) d x
\end{aligned}
$$

Using the Poincaré inequality and the zero-mean condition on $u,(3.46)$ becomes

$$
\begin{aligned}
\frac{d}{d t} \int_{0}^{2 \pi} u^{2}(t) d x & \leq-2\left(\frac{\left(\nu-4 \pi^{2}\right)-\sqrt{16 \pi^{4} \tau\left(\mu K^{2}+16 \pi^{4} \tau K^{4}\right)}}{4 \pi^{2}}\right) \int_{0}^{2 \pi} u^{2}(t) d x \\
& \leq-2 \omega \int_{0}^{2 \pi} u^{2}(t) d x
\end{aligned}
$$

where $\omega$ is defined by (3.24). Solving this inequality, we obtain

$$
\int_{0}^{2 \pi} u^{2}(t) d x \leq e^{-2 \omega t} \int_{0}^{2 \pi} u_{0}^{2}(x, 0) d x, \quad \forall 0 \leq t \leq T_{0} .
$$

From (3.46), we have

$$
\frac{d}{d t} \int_{0}^{2 \pi} u^{2}(t) d x+8 \pi^{2} \omega \int_{0}^{2 \pi} u_{x}^{2}(t) d x \leq 0
$$

Multiplying (3.49) by $e^{\omega t}$, we get

$$
\frac{d}{d t}\left(e^{\omega t} \int_{0}^{2 \pi} u^{2}(t) d x\right)+8 \pi^{2} \omega e^{\omega t} \int_{0}^{2 \pi} u_{x}^{2}(t) d x \leq \omega e^{\omega t} \int_{0}^{2 \pi} u^{2}(t) d x .
$$

Now using (3.48), we get

$$
\frac{d}{d t}\left(e^{\omega t} \int_{0}^{2 \pi} u^{2}(t) d x\right)+8 \pi^{2} \omega e^{\omega t} \int_{0}^{2 \pi} u_{x}^{2}(t) d x \leq \omega e^{-\omega t} \int_{0}^{2 \pi} u_{0}^{2}(x, 0) d x .
$$

Integrating (3.51) from 0 to $T_{0}$, we obtain

$$
\begin{gathered}
\int_{0}^{T_{0}} d\left(e^{\omega t} \int_{0}^{2 \pi} u^{2}(t) d x\right)+8 \pi^{2} \omega \int_{0}^{T_{0}} e^{\omega t} \int_{0}^{2 \pi} u_{x}^{2}(t) d x d t \\
\leq \omega \int_{0}^{T_{0}} e^{-\omega t} \int_{0}^{2 \pi} u_{0}^{2}(x, 0) d x d t
\end{gathered}
$$


which is equivalent to

$$
\begin{aligned}
& e^{\omega T_{0}} \int_{0}^{2 \pi} u^{2}\left(T_{0}\right) d x-\int_{0}^{2 \pi} u_{0}^{2}(x, 0) d x+8 \pi^{2} \omega \int_{0}^{T_{0}} e^{\omega t} \int_{0}^{2 \pi} u_{x}^{2}(t) d x d t \\
& \quad \leq\left(1-e^{-\omega T_{0}}\right) \int_{0}^{2 \pi} u_{0}^{2}(x, 0) d x,
\end{aligned}
$$

which implies

$$
e^{\omega T_{0}} \int_{0}^{2 \pi} u^{2}\left(T_{0}\right) d x+8 \pi^{2} \omega \int_{0}^{T_{o}} e^{\omega t} \int_{0}^{2 \pi} u_{x}^{2}(t) d x d t \leq\left(2-e^{-\omega T_{0}}\right) \int_{0}^{2 \pi} u_{0}^{2}(x, 0) d x
$$

or

$$
\begin{gathered}
8 \pi^{2} \omega \int_{0}^{T_{0}} e^{\omega t} \int_{0}^{2 \pi} u_{x}^{2}(t) d x d t \leq\left(2-e^{-\omega T_{0}}\right) \int_{0}^{2 \pi} u_{0}^{2}(x, 0) d x-e^{\omega T_{0}} \int_{0}^{2 \pi} u^{2}\left(T_{0}\right) d x, \\
\int_{0}^{T_{0}} e^{\omega t} \int_{0}^{2 \pi} u_{x}^{2}(t) d x d t \leq \frac{1}{4 \pi^{2} \omega} \int_{0}^{2 \pi} u_{0}^{2}(x, 0) d x \leq \frac{1}{\omega} \int_{0}^{2 \pi} u_{0}^{2}(x, 0) d x
\end{gathered}
$$

Lemma 3.4. If $\left\|u_{x}(t)\right\| \leq K$, for all $-\tau \leq t<T_{0}$ and $T_{0}=\sup \left\{\delta:\left\|u_{x}(t)\right\| \leq K\right.$ on $\left.0 \leq t \leq \delta\right\}$, then

$$
\int_{0}^{T_{0}} e^{\omega t} \int_{0}^{2 \pi} u_{x}^{2}(t-\tau) d x d t \leq e^{\omega \tau} \int_{-\tau}^{0} \int_{0}^{2 \pi} u_{0_{x}}^{2}(s) d x d s+\frac{e^{\omega \tau}}{\omega} \int_{0}^{2 \pi} u_{0}^{2}(x, 0) d x,
$$

where $\omega$ is defined as in (3.24).

Proof. Consider the term $\int_{0}^{T_{0}} e^{\omega t} \int_{0}^{2 \pi} u_{x}^{2}(t-\tau) d x d t$ and make the change of variable $s=$ $t-\tau$ on that term; we get

$$
\int_{0}^{T_{0}} e^{\omega t} \int_{0}^{2 \pi} u_{x}^{2}(t-\tau) d x d t=\int_{-\tau}^{T_{0}-\tau} e^{\omega(s+\tau)} \int_{0}^{2 \pi} u_{x}^{2}(s) d x d s
$$

Now, since $u(x, s)=u_{0}(x, s)$ in $-\tau \leq s \leq 0$, then $u_{x}(x, s)=u_{0_{x}}(x, s)$, for all $-\tau \leq s \leq 0$. Then the above equality becomes

$$
\int_{0}^{T_{0}} e^{\omega t} \int_{0}^{2 \pi} u_{x}^{2}(t-\tau) d x d t \leq \int_{-\tau}^{0} e^{\omega(s+\tau)} \int_{0}^{2 \pi} u_{0_{x}}^{2}(s) d x d s+\int_{0}^{T_{0}} e^{\omega(s+\tau)} \int_{0}^{2 \pi} u_{x}^{2}(s) d x d s
$$

and since $e^{\omega s} \leq 1$, for $-\tau \leq s \leq 0$, then

$$
\int_{0}^{T_{0}} e^{\omega t} \int_{0}^{2 \pi} u_{x}^{2}(t-\tau) d x d t \leq e^{\omega \tau} \int_{-\tau}^{0} \int_{0}^{2 \pi} u_{0_{x}}^{2}(s) d x d s+e^{\omega \tau} \int_{0}^{T_{0}} e^{\omega s} \int_{0}^{2 \pi} u_{x}^{2}(s) d x d s
$$

Using Lemma 3.3, we obtain the desired result. 
Lemma 3.5 (see [34]). Let $g, h$, and $y$ be three positive and integrable functions on $\left(t_{0}, T\right)$ such that $d y / d t$ is integrable on $\left(t_{0}, T\right)$. If

$$
\frac{d y}{d t} \leq g y+h \quad \forall t_{0} \leq t \leq T
$$

with

$$
\int_{t_{0}}^{T} g(s) d s \leq C_{1}, \quad \int_{t_{0}}^{T} e^{\delta s} h(s) d s \leq C_{2}, \quad \int_{t_{0}}^{T} e^{\delta s} y(s) d s \leq C_{3},
$$

then

$$
y(t) \leq\left[C_{2}+\delta C_{3}+y\left(t_{0}\right)\right] e^{C_{1}} e^{-\delta\left(t-t_{0}\right)}, \quad \forall t_{0} \leq t \leq T,
$$

where $\delta, C_{1}, C_{2}$, and $C_{3}$ are positive constants.

Proof. Multiplying (3.60) by $e^{\delta t}$, we get

$$
\frac{d}{d t}\left(e^{\delta t} y\right)-\delta e^{\delta t} y \leq e^{\delta t} g y+e^{\delta t} h, \quad \text { for } t \geq t_{0}
$$

or

$$
\frac{d}{d t}\left(e^{\delta t} y\right)-g e^{\delta t} y \leq \delta e^{\delta t} y+e^{\delta t} h
$$

Multiplying (3.64) by $\exp \left(-\int_{t_{0}}^{t} g(s) d s\right)$, we obtain

$$
\frac{d}{d t}\left(e^{\delta t} y \exp \left(-\int_{t_{0}}^{t} g(s) d s\right)\right) \leq\left(e^{\delta t} h+\delta e^{\delta t} y\right) \exp \left(-\int_{t_{0}}^{t} g(s) d s\right)
$$

Hence, integrating from $t_{0}$ to $t$, we get

$$
\begin{aligned}
e^{\delta t} y(t) \exp \left(-\int_{t_{0}}^{t} g(s) d s\right)-e^{\delta t_{0}} y\left(t_{0}\right) \leq \int_{t_{0}}^{t}\left(e^{\delta s} h+\delta e^{\delta s} y\right) \exp \left(-\int_{t_{0}}^{s} g(r) d r\right) d s \\
e^{\delta t} y \leq e^{\delta t_{0}} y\left(t_{0}\right) \exp \left(\int_{t_{0}}^{t} g(s) d s\right) \\
+\left[\int_{t_{0}}^{t}\left(e^{\delta s} h+\delta e^{\delta s} y\right) \exp \left(-\int_{t_{0}}^{s} g(r) d r\right) d s\right] \exp \left(\int_{t_{0}}^{t} g(r) d r\right) \\
\leq e^{\delta t_{0}} y\left(t_{0}\right) \exp \left(\int_{t_{0}}^{t} g(s) d s\right)+\int_{t_{0}}^{t}\left(e^{\delta s} h+\delta e^{\delta s} y\right) \exp \left(-\int_{t}^{s} g(r) d r\right) d s \\
\leq e^{\delta t_{0}+C_{1}} y\left(t_{0}\right)+\left(C_{2}+\delta C_{3}\right) e^{C_{1}}
\end{aligned}
$$


or

$$
y \leq\left(C_{2}+\delta C_{3}+y\left(t_{0}\right)\right) e^{C_{1}} e^{-\delta\left(t-t_{0}\right)} .
$$

Theorem 3.6. Let $\tau_{0}=\tau_{0}\left(\mu, u_{0}\right)$, where $\mu=\nu-4 \pi^{2}$ with $\nu>4 \pi^{2}, K$ and $\omega$ are as defined in (3.22), (3.23), and (3.24). For any $\tau<\tau_{0}$ and for any initial condition $u_{0}=u_{0}(x, s) \in$ $C\left([-\tau, 0], H^{1}(0,2 \pi)\right)$, the solution of the generalized Burgers equation (3.4) with periodic boundary conditions satisfies

$$
\left\|u_{x}(t)\right\| \leq \frac{K}{2} e^{-\omega(t / 2)}, \quad \forall t \geq 0
$$

Proof. We use the same method as in [25]. Let

$$
T_{0}=\sup \left\{\delta:\left\|u_{x}(t)\right\| \leq K \text { on } 0 \leq t \leq \delta\right\}
$$

Note that $T_{0}>0$ since $\left\|u_{x}(0)\right\|<K$ and $\left\|u_{x}(t)\right\|$ is continuous. To prove that $T_{0}=+\infty$, we argue by contradiction. For $T_{0}<+\infty$, we have

$$
\begin{gathered}
\left\|u_{x}(t)\right\| \leq K, \quad \forall-\tau \leq t<T_{0}, \\
\left\|u_{x}\left(T_{0}\right)\right\|=K .
\end{gathered}
$$

Using (3.4), we get

$$
\begin{aligned}
\frac{d}{d t} \int_{0}^{2 \pi} u_{x}^{2}(t) d x= & -2 v \int_{0}^{2 \pi} u_{x x}^{2}(t) d x-2 \int_{0}^{2 \pi} u_{x x}(t) u(t) d x \\
& +2 \int_{0}^{2 \pi} u_{x x}(t) u(t-\tau) u_{x}(t) d x
\end{aligned}
$$

By using Cauchy-Schwarz and Young's inequalities simultaneously on (3.71), we obtain

$$
\frac{d}{d t} \int_{0}^{2 \pi} u_{x}^{2}(t) d x \leq \frac{1}{2 v} \int_{0}^{2 \pi} u^{2}(t-\tau) u_{x}^{2}(t) d x-2 \int_{0}^{2 \pi} u_{x x}(t) u(t) d x
$$

Using integration by parts, we get

$$
\frac{d}{d t} \int_{0}^{2 \pi} u_{x}^{2}(t) d x \leq \frac{1}{2 \nu} \int_{0}^{2 \pi} u^{2}(t-\tau) u_{x}^{2}(t) d x+2 \int_{0}^{2 \pi} u_{x}^{2}(t) d x
$$

Since $|u(t-\tau)| \leq(2 \pi)^{2}\left\|u_{x}(t-\tau)\right\|$, then

$$
\frac{d}{d t} \int_{0}^{2 \pi} u_{x}^{2}(t) d x-2 \int_{0}^{2 \pi} u_{x}^{2}(t) d x \leq \frac{8 \pi^{4}}{\nu} \int_{0}^{2 \pi} u_{x}^{2}(t-\tau) d x \int_{0}^{2 \pi} u_{x}^{2}(t) d x
$$


or

$$
\frac{d}{d t}\left(e^{-2 t} \int_{0}^{2 \pi} u_{x}^{2}(t) d x\right) \leq \frac{8 \pi^{4}}{\nu} \int_{0}^{2 \pi} u_{x}^{2}(t-\tau) d x\left(e^{-2 t} \int_{0}^{2 \pi} u_{x}^{2}(t) d x\right)
$$

Applying Lemma 3.5 on (3.75) with

$$
\begin{gathered}
y=e^{-2 t} \int_{0}^{2 \pi} u_{x}^{2}(t) d x, \\
g=\frac{8 \pi^{4}}{\nu}\left(\int_{0}^{2 \pi} u_{x}^{2}(t-\tau) d x\right), \\
h=0, \\
\delta=\omega, \\
C_{1}=\frac{8 \pi^{4}}{\nu}\left(e^{\omega \tau} \int_{-\tau}^{0} \int_{0}^{2 \pi} u_{0_{x}}^{2}(s) d x d s+\frac{e^{\omega \tau}}{\omega} \int_{0}^{2 \pi} u_{0}^{2}(x, 0) d x d s\right) \quad \text { (by Lemma 3.4), } \\
C_{2}=0, \\
C_{3}=\frac{1}{\omega-2} \int_{0}^{2 \pi} u_{0}^{2}(x, 0) d x \quad(\text { by Lemma 3.3), }
\end{gathered}
$$

then we have for $0 \leq t \leq T_{0}$,

$$
\begin{aligned}
\int_{0}^{2 \pi} u_{x}^{2}(t) d x \leq & {\left[0+\omega\left(\frac{1}{\omega-2} \int_{0}^{2 \pi} u_{0}^{2}(x, 0) d x\right)+\int_{0}^{2 \pi} u_{0_{x}}^{2}(x, 0) d x\right] } \\
& \times \exp \left[\frac{8 \pi^{4}}{v}\left(e^{\omega \tau} \int_{-\tau}^{0} \int_{0}^{2 \pi} u_{0_{x}}^{2}(s) d x d s+\frac{e^{\omega \tau}}{\omega} \int_{0}^{2 \pi} u_{0}^{2}(x, 0) d x d s\right)\right] e^{-\omega t}
\end{aligned}
$$

or

$$
\int_{0}^{2 \pi} u_{x}^{2}(t) d x \leq \frac{K^{2}}{4} e^{-\omega t}
$$

which implies that

$$
\left\|u_{x}\left(T_{0}\right)\right\| \leq \frac{K}{2} e^{-\omega\left(T_{0} / 2\right)}
$$

which is in contradiction to our assumption. Therefore, $T_{0}=+\infty$ and then

$$
\left\|u_{x}(t)\right\| \leq \frac{K}{2} e^{-\omega(t / 2)}, \quad \forall t \geq 0
$$




\section{Numerical results}

In this section, we would like to find a Fourier representation for the generalized Burgers equation without a time delay

$$
u_{t}=v u_{x x}-u u_{x}+u+h(x),
$$

and the generalized Burgers equation with a time delay

$$
u_{t}(x, t)=v u_{x x}(x, t)-u(x, t-\tau) u_{x}(x, t)+u(x, t),
$$

with periodic boundary conditions

$$
u(0, t)=u(2 \pi, t)
$$

Equation (4.1) can be written as

$$
\frac{\partial u}{\partial t}=G(u, h)
$$

where $G(u, h)=v u_{x x}-u u_{x}+u+h(x)$.

Spectral approximation could be used to find the Fourier representation because of its accuracy and efficiency since we can expand the function $u$ in terms of an infinite sequence of orthogonal functions $\left\{\Phi_{k}\right\}$,

$$
u=\sum_{k=-\infty}^{\infty} \hat{u}_{k} \Phi_{k}
$$

But since most numerical methods based upon Fourier series cannot be implemented directly by standard treatment of Fourier series because the Fourier coefficients of an arbitrary complex-valued function are not known and must be approximated in some way, we use the discrete Fourier series [8]. That is, for any integer $N>0$, we consider the set of points

$$
x_{j}=\frac{2 \pi j}{N}, \quad j=0, \ldots, N-1 .
$$

The discrete Fourier coefficients of a complex-valued function $u$ in $[0,2 \pi]$ with respect to these points are

$$
\tilde{u}_{k}=\frac{1}{N} \sum_{j=0}^{N-1} u\left(x_{j}, t\right) e^{-i k x_{j}}, \quad-\frac{N}{2} \leq k \leq \frac{N}{2}-1,
$$

where

$$
u(x, t)=\sum_{k=-N / 2}^{N / 2-1} e^{i k x} \hat{u}(k, t) .
$$


Then, by differentiating (4.8) with respect to $x$ and with respect to $t$, we get

$$
\begin{aligned}
& u_{x}(x, t)=i \sum_{k=-N / 2}^{N / 2-1} k e^{i k x} \hat{u}(k, t), \\
& u_{x x}(x, t)=-\sum_{k=-N / 2}^{N / 2-1} k^{2} e^{i k x} \hat{u}(k, t), \\
& u_{t}(x, t)=-\sum_{k=-N / 2}^{N / 2-1} e^{i k x} \hat{u}_{t}(k, t) .
\end{aligned}
$$

If we substitute (4.8), (4.9), (4.10), and (4.11) in (4.1), we get

$$
\begin{aligned}
\hat{u}_{t}(k, t)= & \left(1-v k^{2}\right) \hat{u}(k, t)-i k \sum_{k=p+q} \hat{u}(p, t) \hat{u}(q, t) \\
& -i k \sum_{p+q=k \pm N} \hat{u}(p, t) \hat{u}(q, t)+\hat{h}(k) .
\end{aligned}
$$

Also, substituting (4.8), (4.9), (4.10), and (4.11) in (4.2), we obtain

$$
\begin{aligned}
\hat{u}_{t}(k, t)= & \left(1-v k^{2}\right) \hat{u}(k, t)-i k \sum_{k=p+q} \hat{u}(p, t-\tau) \hat{u}(q, t) \\
& -i k \sum_{p+q=k \pm N} \hat{u}(p, t-\tau) \hat{u}(q, t) .
\end{aligned}
$$

All the nonlinear terms in (4.12) and (4.13) were evaluated in the physical space followed by the discrete Fourier transform to find the Fourier coefficients. The aliasing error was removed by truncation in the manner described in [8], that is, by performing all multiplication in a physical space followed by the discrete Fourier transform to determine the corresponding Fourier coefficients.

Two computer programs that use a spectral Galerkin method with $N=256$ were written to solve both (4.12) and (4.13). The value of $N=256$ in those equations was chosen so that not only the truncation error is kept down to a minimum, but also the aliasing error caused by the nonlinear term is completely removed. The "slaved-frog" scheme was used [18]. That is

$$
u_{n+1}=e^{-2 \alpha \delta t} u_{n-1}+\left(\frac{1-e^{-2 \alpha \delta t}}{\alpha}\right) f_{n}
$$

where $u_{n}=u\left(t_{n}\right), f_{n}=f\left(t_{n}\right)$. This is obtained from the exact relation

$$
u(t+\delta t)=e^{-2 \alpha \delta t} u(t-\delta t)+\int_{t-\delta t}^{t+\delta t} e^{-\alpha(t+\delta t-s)} f(s) d s .
$$

Figure 4.1 presents the steady state solution of the generalized Burgers equation (4.1) without time delay when $h(x)=0.5 \sin (x)$ and $u(x, 0)=\sin (x)$ for different viscosity. One 

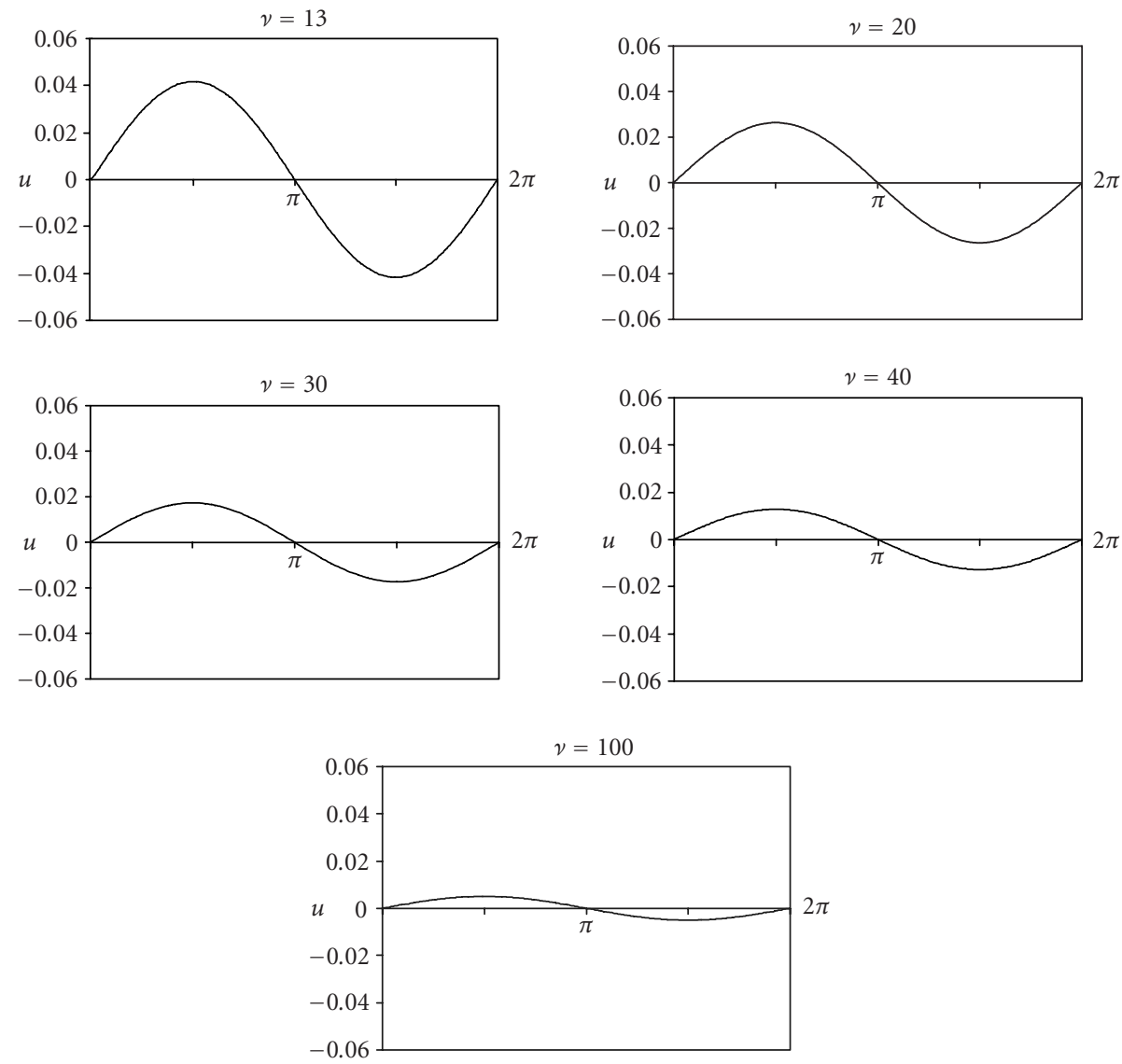

Figure 4.1. Steady state solutions of the generalized Burgers equation without time delay for different values of $v$ with $h(x)=0.5 \sin (x)$ and $u(x, 0)=\sin (x)$.

can observe that when $v$ is large, the solution decays to the steady state quickly. This is because the linear diffusion term is controlled by the viscosity. Hence, for small $\nu$, the convection term will act to sharpen the solution, while the diffusion term will try to smooth it out. This competition of sharpening and smoothing out of solutions will take some time until the solution reaches steady states. But for large $v$, the diffusion term will dominate the equation behavior. As a result, the solution will evolve to the steady state quickly. Other sinusoidal terms for $h(x)$ and $u(x, 0)$ were used and similar results were obtained (see Figure 4.2).

Figures 4.3 and 4.4 present the energy or Lyapunov function curve of solution of (4.2) with different values of delays. It can be seen that for small $\tau$ 's, the energy always decays to zero exponentially. In Figure 4.3, we consider $u(x, s)=10(1+s)(\sin 3 x+\sin 2 x+\sin x)$ and observe that the solution will decay to zero exponentially faster for large values of $\tau$ than for small ones. This is because the energy value of $u(x, s)$ is increasing. However, if we take the case of $u(x, s)=10(1-s)(\sin 3 x+\sin 2 x+\sin x)$ (i.e., the energy value is 

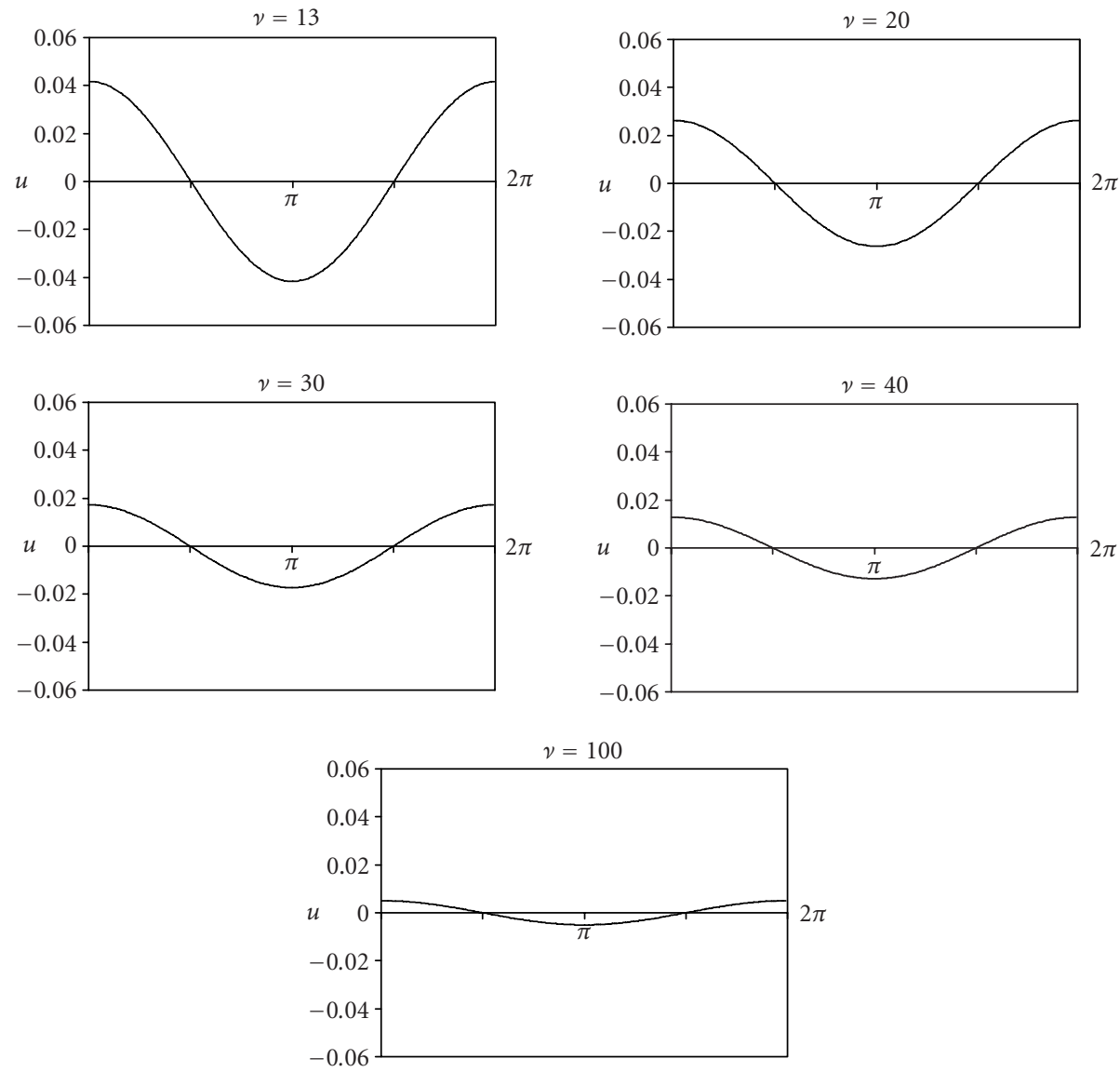

Figure 4.2. Steady state solutions of the generalized Burgers equation without time delay for different values of $\nu$ with $h(x)=0.5 \cos (x)$ and $u(x, 0)=\cos (x)$.

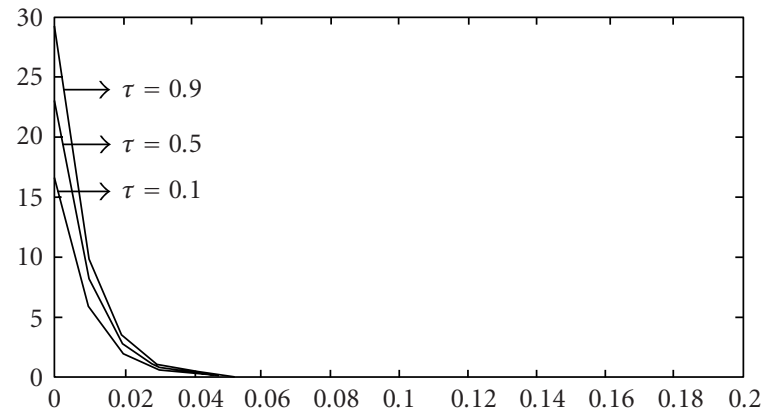

Figure 4.3. Energy curve for the solution of the generalized Burgers equation with time delay for different values of $\tau$ with initial condition $u(x, s)=10(1+s)(\sin 3 x+\sin 2 x+\sin x)$. 


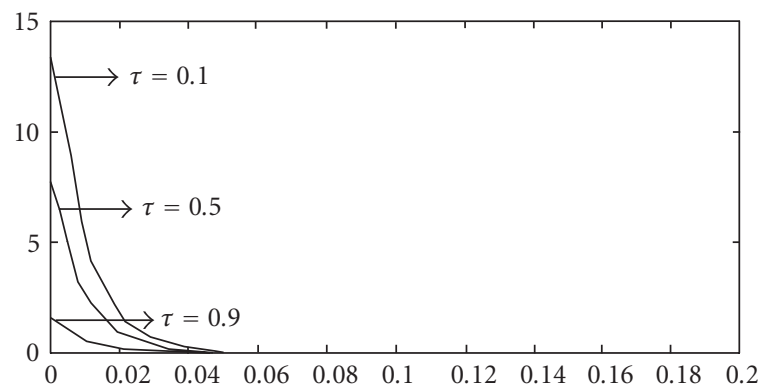

Figure 4.4. Energy curve for the solution of the generalized Burgers equation with time delay for different values of $\tau$ with initial condition $u(x, s)=10(1-s)(\sin 3 x+\sin 2 x+\sin x)$.

decreasing), one can see that the solution decays to zero exponentially quickly when the delay $\tau$ is small (see Figure 4.4). The numerical results obtained are in accordance with the analytical ones presented in Sections 2 and 3.

\section{Concluding remarks}

In this paper, we studied the generalized Burgers equation with periodic boundary conditions on the interval $[0,2 \pi]$ with and without introducing a time delay for sufficiently large viscosity. By using Lyapunov theory, we showed that for the generalized Burgers equation without a time delay and when $h(x)=0$, the equation is globally asymptotically stable. Moreover, we showed that when $h(x) \neq 0$, the steady state solution is bounded and unique. For the generalized Burgers equation with a time delay and when $h(x)=0$, we showed that the equation is exponentially stable under small delays. We presented some numerical results by using the spectral method to support the analytical results given in Sections 2 and 3. The case when $h(x) \neq 0$ in the generalized time-delayed Burgers equation and the analysis of the behavior of its solution for different values of $h(x)$ will be the subject of future studies.

\section{References}

[1] M. J. Ablowitz and S. De Lillo, The Burgers equation under deterministic and stochastic forcing, Phys. D 92 (1996), no. 3-4, 245-259.

[2] G. Avalos, I. Lasiecka, and R. Rebarber, Lack of time-delay robustness for stabilization of a structural acoustics model, SIAM J. Control Optim. 37 (1999), no. 5, 1394-1418.

[3] A. Balogh and M. Krstic, Boundary control of the Korteweg-de Vries-Burgers equation: further results on stabilization and well-posedness, with numerical demonstration, IEEE Trans. Automat. Control 45 (2000), no. 9, 1739-1745.

[4] P. Broadbridge, The forced Burgers equation, plant roots and Schrödinger's eigenfunctions, J. Engrg. Math. 36 (1999), no. 1-2, 25-39.

[5] J. M. Burgers, A mathematical model illustrating the theory of turbulence, Advances in Applied Mechanics, Academic Press, New York, 1948, pp. 171-199.

[6] The Nonlinear Diffusion Equation, Reidel, Massachusetts, 1974.

[7] J. Burns, A. Balogh, D. S. Gilliam, and V. I. Shubov, Numerical stationary solutions for a viscous Burgers' equation, J. Math. Systems Estim. Control 8 (1998), no. 2, 1-16. 
[8] C. Canuto, M. Y. Hussaini, A. Quarteroni, and T. A. Zang, Spectral Methods in Fluid Dynamics, Springer Series in Computational Physics, Springer-Verlag, New York, 1988.

[9] H. Choi, R. Temam, P. Moin, and J. Kim, Feedback control for unsteady flow and its application to the stochastic Burgers equation, J. Fluid Mech. 253 (1993), 509-543.

[10] R. Datko, Not all feedback stabilized hyperbolic systems are robust with respect to small time delays in their feedbacks, SIAM J. Control Optim. 26 (1988), no. 3, 697-713.

[11] R. Datko, J. Lagnese, and M. P. Polis, An example on the effect of time delays in boundary feedback stabilization of wave equations, SIAM J. Control Optim. 24 (1986), no. 1, 152-156.

[12] R. Datko and Y. C. You, Some second-order vibrating systems cannot tolerate small time delays in their damping, J. Optim. Theory Appl. 70 (1991), no. 3, 521-537.

[13] P. P. N. de Groen and G. E. Karadzhov, Slow travelling waves on a finite interval for Burgers'-type equations, J. Comput. Appl. Math. 132 (2001), no. 1, 155-189.

[14] L. A. F. de Oliveira, Instability of homogeneous periodic solutions of parabolic-delay equations, J. Differential Equations 109 (1994), no. 1, 42-76.

[15] A. Dermoune, S. Hamadène, and Y. Ouknine, Limit theorem for the statistical solution of Burgers equation, Stochastic Process. Appl. 81 (1999), no. 2, 217-230.

[16] S. Engelberg, An analytical proof of the linear stability of the viscous shock profile of the Burgers equation with fourth-order viscosity, SIAM J. Math. Anal. 30 (1999), no. 4, 927-936.

[17] G. Friesecke, Exponentially growing solutions for a delay-diffusion equation with negative feedback, J. Differential Equations 98 (1992), no. 1, 1-18.

[18] U. Frisch, Z.-S. She, and O. Thual, Viscoelastic behaviour of cellular solutions to the KuramotoSivashinsky model, J. Fluid Mech. 168 (1986), 221-240.

[19] K. Ito and S. Kang, A dissipative feedback control synthesis for systems arising in fluid dynamics, SIAM J. Control Optim. 32 (1994), no. 3, 831-854.

[20] K. Ito and Y. Yan, Viscous scalar conservation law with nonlinear flux feedback and global attractors, J. Math. Anal. Appl. 227 (1998), no. 1, 271-299.

[21] H. R. Jauslin, H. O. Kreiss, and J. Moser, On the forced Burgers equation with periodic boundary conditions, Differential Equations: La Pietra 1996 (Florence), Proc. Sympos. Pure Math., vol. 65, American Mathematical Society, Rhode Island, 1999, pp. 133-153.

[22] G. Karch, Self-similar large time behavior of solutions to Korteweg-de Vries-Burgers equation, Nonlinear Anal., Ser. A: Theory Methods 35 (1999), no. 2, 199-219.

[23] T. Kobayashi, Adaptive regulator design of a viscous Burgers' system by boundary control, IMA J. Math. Control Inform. 18 (2001), no. 3, 427-437.

[24] M. Krstic, On global stabilization of Burgers' equation by boundary control, Systems Control Lett. 37 (1999), no. 3, 123-141.

[25] W.-J. Liu, Asymptotic behavior of solutions of time-delayed Burgers' equation, Discrete Contin. Dyn. Syst. Ser. B 2 (2002), no. 1, 47-56.

[26] W.-J. Liu and M. Krstic, Backstepping boundary control of Burgers' equation with actuator dynamics, Systems Control Lett. 41 (2000), no. 4, 291-303.

[27] H. V. Ly, K. D. Mease, and E. S. Titi, Distributed and boundary control of the viscous Burgers' equation, Numer. Funct. Anal. Optim. 18 (1997), no. 1-2, 143-188.

[28] S. M. Oliva, Reaction-diffusion equations with nonlinear boundary delay, J. Dynam. Differential Equations 11 (1999), no. 2, 279-296.

[29] Z. Rakib and G. I. Sivashinsky, Instabilities in upward propagating flames, Combust. Sci. Technol. 54 (1987), 69-84.

[30] A. I. Saichev and W. A. Woyczynski, Density fields in Burgers and KdV-Burgers turbulence, SIAM J. Appl. Math. 56 (1996), no. 4, 1008-1038.

[31] N. Smaoui, Analyzing the dynamics of the forced Burgers equation, J. Appl. Math. Stochastic Anal. 13 (2000), no. 3, 269-285. 


\section{Generalized Burgers equation}

[32] N. Smaoui and F. Belgacem, Connections between the convective diffusion equation and the forced Burgers equation, J. Appl. Math. Stochastic Anal. 15 (2002), no. 1, 57-75.

[33] X. Sun and M. J. Ward, Metastability for a generalized Burgers equation with applications to propagating flame fronts, European J. Appl. Math. 10 (1999), no. 1, 27-53.

[34] R. Temam, Infinite-Dimensional Dynamical Systems in Mechanics and Physics, 2nd ed., Applied Mathematical Sciences, vol. 68, Springer-Verlag, New York, 1997.

Nejib Smaoui: Department of Mathematics and Computer Science, Kuwait University, P.O. Box 5969, Safat 13060, Kuwait

E-mail address: smaoui@mcs.sci.kuniv.edu.kw

Mona Mekkaoui: Department of Mathematics and Computer Science, Kuwait University, P.O. Box 5969, Safat 13060, Kuwait 


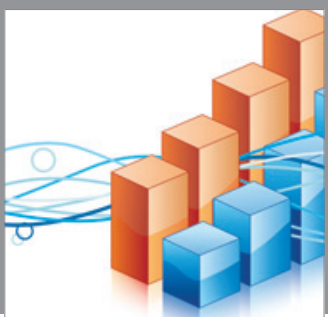

Advances in

Operations Research

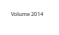

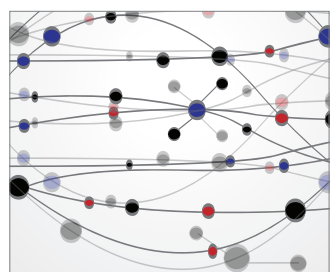

\section{The Scientific} World Journal
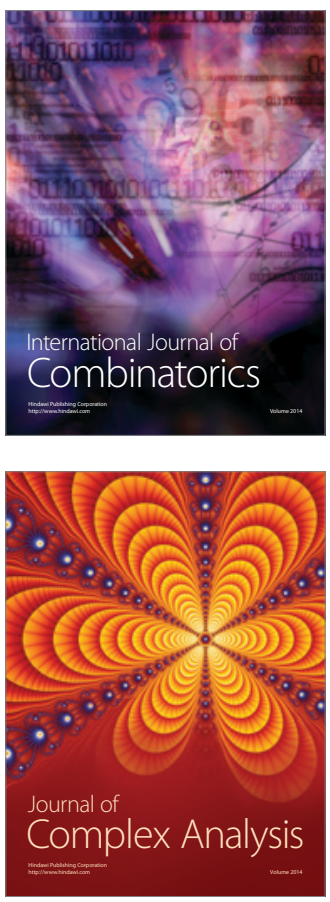

International Journal of

Mathematics and

Mathematical

Sciences
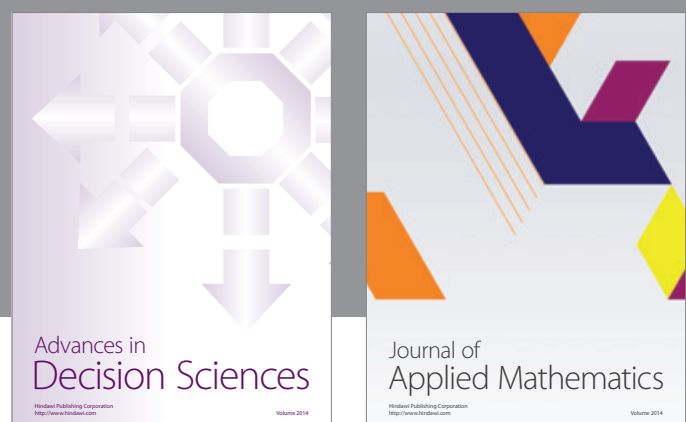

Journal of

Applied Mathematics
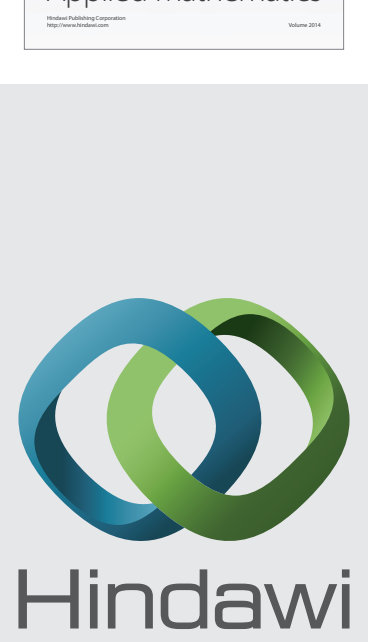

Submit your manuscripts at http://www.hindawi.com
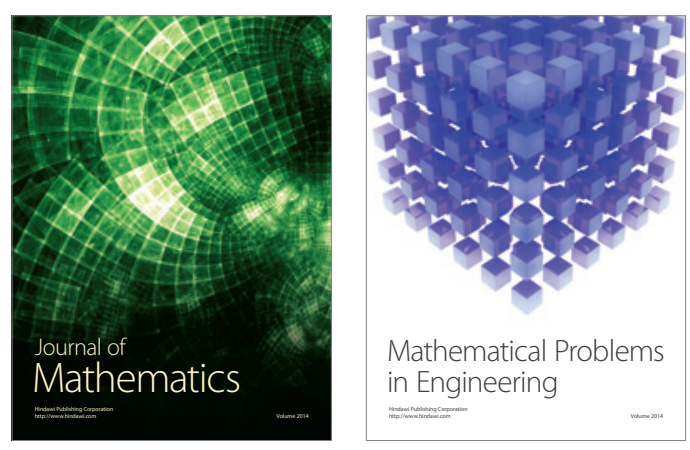

Mathematical Problems in Engineering
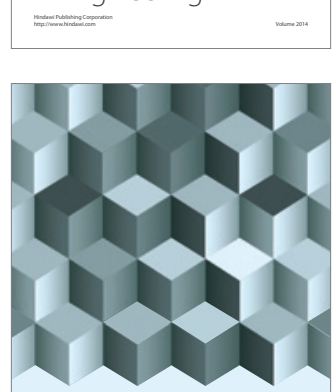

Journal of

Function Spaces
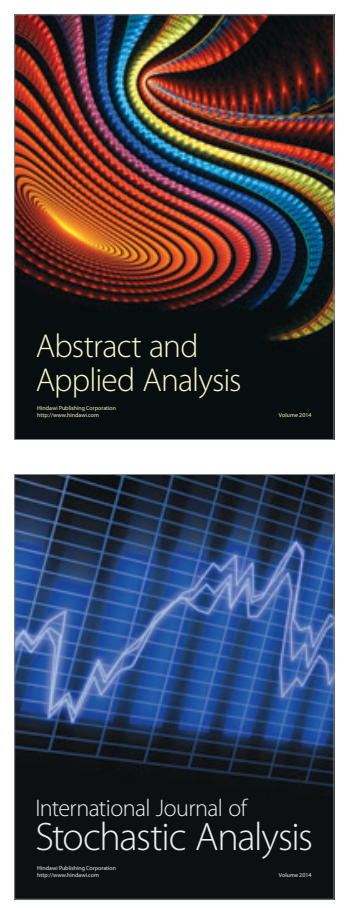

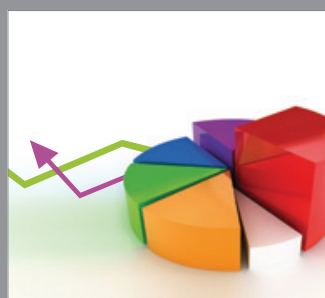

ournal of

Probability and Statistics

Promensencen
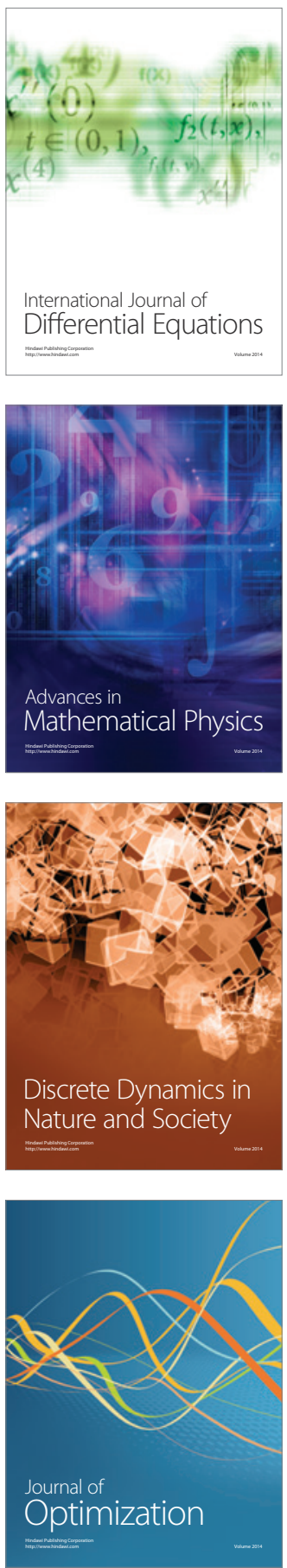